\title{
Optimal control for evolutionary imperfect transmission problems
}

\author{
Luisa Faella ${ }^{1}$ and Carmen Perugia ${ }^{2 *}$
}

"Correspondence:

cperugia@unisannio.it

${ }^{2}$ Dipartimento di Scienze e

Tecnologie, Universitá del Sannio,

Via Dei Mulini 59/A Palazzo

Inarcassa, Benevento, 82100, Italia

Full list of author information is

available at the end of the article

\begin{abstract}
We study the optimal control problem of a second order linear evolution equation defined in two-component composites with $\varepsilon$-periodic disconnected inclusions of size $\varepsilon$ in presence of a jump of the solution on the interface that varies according to a parameter $\gamma$. In particular here the case $\gamma<1$ is analyzed. The optimal control theory, introduced by Lions (Optimal Control of System Governed by Partial Differential Equations, 1971), leads us to characterize the control as the solution of a set of equations, called optimality conditions. The main result of this paper proves that the optimal control of the $\varepsilon$-problem, which is the unique minimum point of a quadratic cost functional $J_{\varepsilon}$, converges to the optimal control of the homogenized problem with respect to a suitable limit cost functional $J_{\infty}$. The main difficulties are to find the appropriate limit functional for the control of the homogenized system and to identify the limit of the controls.
\end{abstract}

MSC: Primary 49J20; 35B37; 35B27

Keywords: homogenization; optimal control; evolution equations

\section{Introduction}

In this paper we study the optimal control of a linear hyperbolic problem with oscillating coefficients on a domain $\Omega$ of $\mathbb{R}^{n}$ made up of two components, a connected one $\Omega_{1 \varepsilon}$ and a second one $\Omega_{2 \varepsilon}$, which is the union of $\varepsilon$-periodic disconnected inclusions of size $\varepsilon$. On the interface $\Gamma^{\varepsilon}=\partial \Omega_{2 \varepsilon}$ separating the two components, we prescribe the continuity of the conormal derivatives and a jump of the solution proportional to the conormal derivatives through a function of order $\varepsilon^{\gamma}$, meanwhile, a Dirichlet condition is imposed on the exterior boundary $\partial \Omega$ (see Figure 1). The order of magnitude of this parameter, with respect to the period $\varepsilon$, determines the influence of the contact barrier in the propagation properties of the medium. Indeed this problem models the wave propagation in a medium made up of two components with very different coefficients of propagation, which gives rise to a jump in the boundary condition on the interface. This interface condition is the mathematical interpretation of imperfect interface characterized by the discontinuity of the displacement (see [1-10] and references therein).

This work connects the corresponding homogenization and correctors results proved respectively in [5] and [6]. The first question of this paper deals with the existence of an optimal control of the $\varepsilon$-problem with respect to a quadratic functional. If such a control exists, the second and more interesting question is: does the optimal control of the $\varepsilon$-problem converge as $\varepsilon \rightarrow 0$ to the optimal control of the homogenized problem with 
Figure $1 \Omega_{\varepsilon}$.

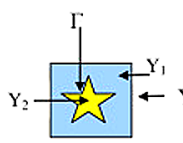

$\mathrm{y}=\mathrm{x} / \varepsilon$

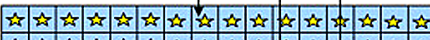

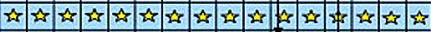

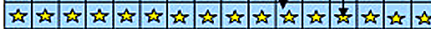

स्र

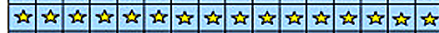

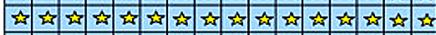

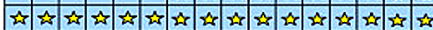

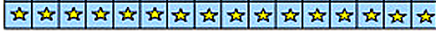

respect to a suitable cost functional? The optimal control of one or more aspects of a problem entails the minimization of a cost functional which describes physical quantities involved in the specific problem. To give a positive answer to both questions, we refer to the techniques used by Lions in [11]. These ones consist of the construction of the adjoint problem and the research of a set of equations, called optimality conditions, characterizing the optimal control and the related cost functional. As already shown by Hummel in [12], for the homogenization results in the elliptic case, one cannot expect to have boundedness of the solutions when $\gamma>1$. Hence it would be natural here to suppose $\gamma \leq 1$. Nevertheless in this paper we analyze the case $\gamma<1$ being the case $\gamma=1$ more delicate. Indeed it is already known from previous studies (see [5]) that the asymptotic behavior of the $\varepsilon$ problem differs in terms of the homogenized problems in the two cases $\gamma<1$ and $\gamma=1$. The second one is the most complicated one, since the limit problem is a coupled system of a P.D.E. and a O.D.E. and gives rise to what is called a memory effect. When searching an optimal control result for the case $\gamma=1$ we cannot adapt the same arguments used for the more general case $\gamma<1$. In fact the homogenized problem is no more symmetric, hence the adjoint of the homogenized problem does not coincide with the limit of the adjoint problem at $\varepsilon$-level. We will use other techniques to study the case $\gamma=1$.

The plan of the paper is as follows.

In Section 2 we recall some useful properties of a specific functional space, introduced in $[9,13]$ by Donato and Monsurrò in the elliptic framework, suitable for the solutions of this kind of interface problems. Successively, we recall some further properties, involving evolution triples, needed in the time-dependent framework. They have been proved by Donato et al. in [5].

In Section 3, we state the main result, Theorem 2.1, whose proof is performed into several steps. At first we describe the homogenization result for the hyperbolic problem; we refer the reader also to [5] where all the proofs can be found. Then we study the control problem. Our approach to the optimal control problem for a hyperbolic equation consists in applying Pontryagin maximum principle to obtain the expression for the optimal control $w_{\varepsilon}$ at level $\varepsilon$ in terms of the adjoint state $p_{\varepsilon}$, solution of the dual problem. We identify the problem satisfied by the limit $(u, w)$ of the sequence of optimal pairs $\left\{\left(u_{\varepsilon}, w_{\varepsilon}\right)\right\}_{\varepsilon}$, where $u_{\varepsilon}$ denotes the state of the system to be controlled, and also the problem satisfied by the limit $p$ of $\left\{p_{\varepsilon}\right\}_{\varepsilon}$. We observe that $p$ is the adjoint state corresponding to an optimal control problem for the limit equation and, by the maximum principle, we infer that the pair $(u, w)$ is optimal. The indirect approach, using the adjoint problems and the maximum principle, is necessary since the convergences occur only in $L^{2}$-weak topology and not, as usual in homogenization theory, in $L^{2}$-strong topology. The crucial point is to obtain compactness 
properties for the sequence of the optimal controls $w_{\varepsilon}$. Finally, we prove that the limit of the minimum points of the cost functional $J_{\varepsilon}$ at level $\varepsilon$ is the minimum point of an appropriate limit cost functional $J_{\infty}$. Let us point out that the cost functional $J_{\infty}$ describe the physical properties of the wave equation for a composite occupying the whole $\Omega$, without any interface. Moreover, we observe that, without any technical difficulties, we can obtain the same optimal control result with a more general quadratic cost functional.

Optimal control problems and the exact controllability in domains with highly oscillating boundary are considered in [14-20]. Moreover, we refer to [21] for control of hyperbolic problems with oscillating coefficients in a fixed domain and to [22, 23] for control of hyperbolic problems in perforated domains.

In [7] and [8] the authors study, respectively, the approximate control and the correctors for a class of parabolic equations with interfacial contact resistance. For the sake of completeness, we recall also [4] where all the results for transmission problems in the elliptic case are collected and $[24,25]$ where the authors treated the homogenization in other types of perforated domains. For the study of similar problems, where the same jump condition is taken into account, we quote here also $[1,2,12,26-30]$ and the references therein.

\section{Statement of the problem and main result}

Let $\Omega$ be an open bounded subset of $\mathbb{R}^{n}(n \geq 2)$ and $\left.Y=\right] 0, l_{1}[\times \cdots \times] 0, l_{n}[$ the reference cell.

We denote by $Y_{1}$ and $Y_{2}$ two nonempty open and disjoint subsets of $Y$ such that

$$
Y=Y_{1} \cup \overline{Y_{2}}
$$

with $Y_{1}$ connected and $\Gamma:=\partial Y_{2}$ of class $C^{2}$. For any $k \in Z^{n}$ we define the translated sets $Y_{i}^{k}$ and $\Gamma_{k}$ as follows:

$$
Y_{i}^{k}:=k_{l}+Y_{i}, \quad \Gamma_{k}:=k_{l}+\Gamma
$$

where $k_{l}=\left(k_{1} l_{1}, \ldots, k_{n} l_{n}\right)$ and $i=1,2$. Let $\{\varepsilon\}$ be a sequence of positive real numbers converging to zero and for any given $\varepsilon$ let us set

$$
K_{\varepsilon}:=\left\{k \in Z^{n} \mid \varepsilon \Gamma_{k} \cap \Omega \neq \emptyset\right\} .
$$

Then we define the two components of $\Omega$ and the interface respectively as follows:

$$
\Omega_{i \varepsilon}:=\Omega \cap\left\{\bigcup_{k \in K_{\varepsilon}} \varepsilon Y_{i}^{k}\right\}, \quad i=1,2, \quad \text { and } \quad \Gamma^{\varepsilon}:=\partial \Omega_{2 \varepsilon} .
$$

We assume that

$$
\partial \Omega \cap\left(\bigcup_{k \in Z^{n}}\left(\varepsilon \Gamma_{k}\right)\right):=\emptyset .
$$

We explicitly observe that by construction, the set $\Omega$ is decomposed into two components $\Omega=\Omega_{1 \varepsilon} \cup \overline{\Omega_{2 \varepsilon}}$ where $\Omega_{1 \varepsilon}$ is a connected set while $\Omega_{2 \varepsilon}$ is a disconnected union of $\varepsilon$-periodic 
disjoint translated sets of $\varepsilon Y_{2}$. Moreover, $\Gamma_{\varepsilon}$ is the interface separating the two components with $\partial \Omega \cup \Gamma_{\varepsilon}=\emptyset$ (see Figure 1).

In the sequel we denote by

- the zero extension to the whole of $\Omega$ of functions defined on $\Omega_{1 \varepsilon}$ or $\Omega_{2 \varepsilon}$;

- $\chi_{E}$ the characteristic function of any measurable set $E \in \mathbb{R}^{n}$;

- $m_{\omega}(v)=\frac{1}{\omega} \int_{\omega} v d x$ the average on $Y$ of any function $v \in L^{1}(\omega)$.

Let us recall (see for instance [31]) that as $\varepsilon \rightarrow 0$, for $i=1,2$,

$$
\chi_{\Omega_{i \varepsilon}} \rightarrow \theta_{i}:=\frac{\left|Y_{i}\right|}{|Y|} \quad \text { weakly in } L^{2}(\Omega)
$$

$\theta_{i}$ being the proportion of the material occupying $\Omega_{i \varepsilon}$.

For any $\varepsilon>0$, let us introduce he functional space $V^{\varepsilon}$ as

$$
V^{\varepsilon}:=\left\{v_{1} \in H^{1}\left(\Omega_{1 \varepsilon}\right) \mid v_{1}=0 \text { on } \partial \Omega\right\},
$$

which is a Banach space if endowed with the norm

$$
\left\|\nu_{1}\right\|_{V^{\varepsilon}}:=\left\|\nabla v_{1}\right\|_{L^{2}\left(\Omega_{1 \varepsilon}\right)}
$$

Clearly, since we do not assume any regularity on $\partial \Omega$, the condition on $\partial \Omega$ in the definition of $V^{\varepsilon}$ has to be understood in a density sense. To be more precise, $V^{\varepsilon}$ is the closure, with respect to the $H^{1}\left(\Omega_{1 \varepsilon}\right)$-norm, of the set of the functions in $C^{\infty}\left(\Omega_{1 \varepsilon}\right)$ with a compact support contained in $\Omega$. This can be done in view of (2.1).

For any $\varepsilon>0$, we set

$$
\begin{gathered}
W^{\varepsilon}:=\left\{v=\left(v_{1}, v_{2}\right) \in L^{2}\left(0, T ; V^{\varepsilon}\right) \times L^{2}\left(0, T ; H^{1}\left(\Omega_{2 \varepsilon}\right)\right) \mid\right. \\
\left.v^{\prime} \in L^{2}\left(0, T ; L^{2}\left(\Omega_{1 \varepsilon}\right)\right) \times L^{2}\left(0, T ; L^{2}\left(\Omega_{2 \varepsilon}\right)\right)\right\},
\end{gathered}
$$

which is a Hilbert space if equipped with the norm

$$
\|v\|_{W^{\varepsilon}}=\left\|v_{1}\right\|_{L^{2}\left(0, T ; V^{\varepsilon}\right)}+\left\|v_{2}\right\|_{L^{2}\left(0, T ; H^{1}\left(\Omega_{2 \varepsilon}\right)\right)}+\left\|v_{1}^{\prime}\right\|_{L^{2}\left(0, T ; L^{2}\left(\Omega_{1 \varepsilon}\right)\right)}+\left\|v_{2}^{\prime}\right\|_{L^{2}\left(0, T ; L^{2}\left(\Omega_{2 \varepsilon}\right)\right)} .
$$

Let $A$ be a $n \times n Y$-periodic matrix field with coefficients in $L^{\infty}(Y)$ such that for any $\lambda \in \mathbb{R}^{n}$ and a.e. in $Y$ one has

$$
\left\{\begin{array}{l}
(A(x) \lambda, \lambda) \geq \alpha|\lambda|^{2}, \\
|A(x) \lambda| \leq \beta|\lambda| \\
a_{i, j}=a_{j, i} \quad \text { for every } 1 \leq i, j \leq n,
\end{array}\right.
$$

with $0<\alpha<\beta$.

Moreover, we suppose that $h$ is a $Y$-periodic function such that

$$
h \in L^{\infty}(\Gamma) \text { and } \exists h_{0} \in \mathbb{R} \text { such that } 0<h_{0}<h(y) \text { a.e. in } \Gamma \text {. }
$$

For any $\varepsilon>0$, we set

$$
h^{\varepsilon}(x):=h\left(\frac{x}{\varepsilon}\right)
$$


and

$$
A^{\varepsilon}(x):=A(x / \varepsilon) \text {. }
$$

The aim of this paper is to study the optimal control and its asymptotic behavior as $\varepsilon \rightarrow 0$ for a hyperbolic imperfect transmission problem defined in the domain $\Omega$ previously described.

More precisely let $z_{\varepsilon}=\left(z_{1 \varepsilon}, z_{2 \varepsilon}\right)$ be a control to be found in $L^{2}\left(0, T ; L^{2}\left(\Omega_{1 \varepsilon}\right)\right) \times L^{2}(0, T$; $\left.L^{2}\left(\Omega_{2 \varepsilon}\right)\right)$. For any fixed $T>0$ let us consider the following problem:

$$
\left\{\begin{array}{l}
\left.u_{1 \varepsilon}^{\prime \prime}-\operatorname{div}\left(A^{\varepsilon} \nabla u_{1 \varepsilon}\right)=f_{1 \varepsilon}+z_{1 \varepsilon} \quad \text { in } \Omega_{1 \varepsilon} \times\right] 0, T[, \\
\left.u_{2 \varepsilon}^{\prime \prime}-\operatorname{div}\left(A^{\varepsilon} \nabla u_{2 \varepsilon}\right)=f_{2 \varepsilon}+z_{2 \varepsilon} \quad \text { in } \Omega_{2 \varepsilon} \times\right] 0, T[, \\
\left.A^{\varepsilon} \nabla u_{1 \varepsilon} \cdot n_{1 \varepsilon}=-A^{\varepsilon} \nabla u_{2 \varepsilon} \cdot n_{2 \varepsilon} \quad \text { on } \Gamma^{\varepsilon} \times\right] 0, T[, \\
\left.A^{\varepsilon} \nabla u_{1 \varepsilon} \cdot n_{1 \varepsilon}=-\varepsilon^{\gamma} h^{\varepsilon}\left(u_{1 \varepsilon}-u_{2 \varepsilon}\right) \quad \text { on } \Gamma^{\varepsilon} \times\right] 0, T[, \\
\left.u_{1 \varepsilon}=0 \quad \text { on } \partial \Omega \times\right] 0, T[, \\
u_{1 \varepsilon}(0)=U_{1 \varepsilon}^{0} \quad \text { in } \Omega_{1 \varepsilon}, \quad u_{2 \varepsilon}(0)=U_{2 \varepsilon}^{0} \quad \text { in } \Omega_{2 \varepsilon}, \\
u_{1 \varepsilon}^{\prime}(0)=U_{1 \varepsilon}^{1} \quad \text { in } \Omega_{1 \varepsilon}, \quad u_{2 \varepsilon}^{\prime}(0)=U_{2 \varepsilon}^{1} \quad \text { in } \Omega_{2 \varepsilon},
\end{array}\right.
$$

where $\gamma<1, n_{i \varepsilon}$ is the unitary outward normal to $\Omega_{i \varepsilon}, i=1,2$, and

$$
\left\{\begin{array}{l}
\text { (i) } f_{\varepsilon}=\left(f_{1 \varepsilon}, f_{2 \varepsilon}\right) \in L^{2}\left(0, T ; L^{2}\left(\Omega_{1 \varepsilon}\right)\right) \times L^{2}\left(0, T ; L^{2}\left(\Omega_{2 \varepsilon}\right)\right), \\
\text { (ii) } U_{\varepsilon}^{0}=\left(U_{1 \varepsilon}^{0}, U_{2 \varepsilon}^{0}\right) \in V^{\varepsilon} \times H^{1}\left(\Omega_{2 \varepsilon}\right), \\
\text { (iii) } \quad U_{\varepsilon}^{1}=\left(U_{1 \varepsilon}^{1}, U_{2 \varepsilon}^{1}\right) \in L^{2}\left(\Omega_{1 \varepsilon}\right) \times L^{2}\left(\Omega_{2 \varepsilon}\right) .
\end{array}\right.
$$

Let us introduce a class of function spaces expressly considered for the solution of this particular kind of interface problems. They were defined for the first time in [3,32] and [12] in the framework of the study of the analogous elliptic problem. Clearly, the space of the solutions must take into account either the geometry of the domain in which the material is confined or the boundary and interfacial conditions. For every $\gamma \in \mathbb{R}$, we set (see also [9])

$$
H_{\gamma}^{\varepsilon}:=\left\{v=\left(v_{1}, v_{2}\right) \mid v_{1} \in V^{\varepsilon} \text { and } v_{2} \in H^{1}\left(\Omega_{2 \varepsilon}\right)\right\}
$$

The space $H_{\gamma}^{\varepsilon}$ is a Banach space when equipped with the norm

$$
\|v\|_{H_{\gamma}^{\varepsilon}}^{2}:=\left\|\nabla v_{1}\right\|_{L^{2}\left(\Omega_{1 \varepsilon}\right)}^{2}+\left\|\nabla v_{2}\right\|_{L^{2}\left(\Omega_{2 \varepsilon}\right)}^{2}+\varepsilon^{\gamma}\left\|v_{1}-v_{2}\right\|_{L^{2}\left(\Gamma^{\varepsilon}\right)}^{2} .
$$

It is easy to check that, if $0<\varepsilon<1$ and $\gamma_{1} \leq \gamma_{2}$, then

$$
\|v\|_{H_{\gamma_{2}}^{\varepsilon}} \leq\|v\|_{H_{\gamma_{1}}^{\varepsilon}}
$$

Moreover, for every fixed $\varepsilon$ the norms of $H_{\gamma}^{\varepsilon}$ and $V^{\varepsilon} \times H^{1}\left(\Omega_{2 \varepsilon}\right)$ are equivalent; see [13] for details.

We point out that $H_{\gamma}^{\varepsilon}$ is a separable and reflexive Banach space dense in $L^{2}\left(\Omega_{1 \varepsilon}\right) \times$ $L^{2}\left(\Omega_{2 \varepsilon}\right)$. Moreover, $H_{\gamma}^{\varepsilon} \subseteq L^{2}\left(\Omega_{1 \varepsilon}\right) \times L^{2}\left(\Omega_{2 \varepsilon}\right)$ with continuous imbedding. On the other hand, one sees that $L^{2}\left(\Omega_{1 \varepsilon}\right) \times L^{2}\left(\Omega_{2 \varepsilon}\right) \subseteq\left(H_{\gamma}^{\varepsilon}\right)^{\prime}$, with $L^{2}\left(\Omega_{1 \varepsilon}\right) \times L^{2}\left(\Omega_{2 \varepsilon}\right)$ separable Hilbert 
space. This means that the triple $\left(H_{\gamma}^{\varepsilon}, L^{2}\left(\Omega_{1 \varepsilon}\right) \times L^{2}\left(\Omega_{2 \varepsilon}\right),\left(H_{\gamma}^{\varepsilon}\right)^{\prime}\right)$ is an evolution triple. We refer the reader to [5] for an in-depth analysis on this aspect. By using an approach to the evolutionary problems based on evolution triples, as far as the weak formulation of problem (2.8) is concerned, we assume as precise formulation of formal problem the following one (see [18]):

$$
\left\{\begin{array}{l}
\text { find } u_{\varepsilon}=\left(u_{1 \varepsilon}, u_{2 \varepsilon}\right) \text { in } W^{\varepsilon} \text { such that } \\
\begin{array}{lll}
\left\langle u_{1 \varepsilon}^{\prime \prime}, v_{1}\right\rangle_{\left(V^{\varepsilon}\right)^{\prime}, V^{\varepsilon}}+\left\langle u_{2 \varepsilon}^{\prime \prime}, v_{2}\right\rangle_{\left(H^{1}\left(\Omega_{2 \varepsilon}\right)\right)^{\prime}, H^{1}\left(\Omega_{2 \varepsilon}\right)} \\
\quad+\int_{\Omega_{1 \varepsilon}} A^{\varepsilon} \nabla u_{1 \varepsilon} \cdot \nabla v_{1} d x+\int_{\Omega_{2 \varepsilon}} A^{\varepsilon} \nabla u_{2 \varepsilon} \cdot \nabla v_{2} d x \\
\quad+\varepsilon^{\gamma} \int_{\Gamma^{\varepsilon}} h^{\varepsilon}\left(u_{1 \varepsilon}-u_{2 \varepsilon}\right)\left(v_{1}-v_{2}\right) d \sigma_{x}=\int_{\Omega_{1 \varepsilon}}\left(f_{1 \varepsilon}+z_{1 \varepsilon}\right) v_{1} d x \\
\quad+\int_{\Omega_{2 \varepsilon}}\left(f_{2 \varepsilon}+z_{2 \varepsilon}\right) v_{2} d x & \forall\left(v_{1}, v_{2}\right) \in V^{\varepsilon} \times H^{1}\left(\Omega_{2 \varepsilon}\right) \text { in } \mathcal{D}^{\prime}(0, T), \\
u_{1 \varepsilon}(0)=U_{1 \varepsilon}^{0} & \text { in } \Omega_{1 \varepsilon}, \quad u_{2 \varepsilon}(0)=U_{2 \varepsilon}^{0} \quad \text { in } \Omega_{2 \varepsilon} \\
u_{1 \varepsilon}^{\prime}(0)=U_{1 \varepsilon}^{1} & \text { in } \Omega_{1 \varepsilon}, \quad u_{2 \varepsilon}^{\prime}(0)=U_{2 \varepsilon}^{1} & \text { in } \Omega_{2 \varepsilon} .
\end{array}
\end{array}\right.
$$

An abstract Galerkin method provides the existence and uniqueness result for the solution of problem (2.10) for any $\varepsilon>0$ and also some $a$ priori estimates (see [5]). We point out that the unique solution $u_{\varepsilon}\left(z_{\varepsilon}\right)$ of problem (2.10) is said the 'state' of the system to be controlled and (2.10) are called 'state equations'.

To any control $z_{\varepsilon} \in L^{2}\left(0, T ; L^{2}\left(\Omega_{1 \varepsilon}\right)\right) \times L^{2}\left(0, T ; L^{2}\left(\Omega_{2 \varepsilon}\right)\right)$ we associate the cost functional $J_{\varepsilon}: L^{2}\left(0, T ; L^{2}\left(\Omega_{1 \varepsilon}\right) \times L^{2}\left(\Omega_{2 \varepsilon}\right)\right) \rightarrow \mathbb{R}$ defined in the following way:

$$
\begin{aligned}
J_{\varepsilon}\left(z_{\varepsilon}\right):= & \frac{1}{2} \int_{0}^{T} \int_{\Omega_{1 \varepsilon}}\left|u_{1 \varepsilon}\left(z_{\varepsilon}\right)\right|^{2}+\frac{1}{2} \int_{0}^{T} \int_{\Omega_{2 \varepsilon}}\left|u_{2 \varepsilon}\left(z_{\varepsilon}\right)\right|^{2} \\
& +\frac{1}{2} \int_{0}^{T} \int_{\Omega_{1 \varepsilon}}\left|z_{1 \varepsilon}\right|^{2}+\frac{1}{2} \int_{0}^{T} \int_{\Omega_{2 \varepsilon}}\left|z_{2 \varepsilon}\right|^{2} .
\end{aligned}
$$

This functional is continuous, strictly convex and coercive. Hence, by applying the direct method in the calculus of variations, the following minimum problem:

$$
\min \left\{J_{\varepsilon}(z): z \in L^{2}\left(0, T ; L^{2}\left(\Omega_{1 \varepsilon}\right) \times L^{2}\left(\Omega_{2 \varepsilon}\right)\right)\right\}
$$

admits a unique solution $w_{\varepsilon}$ which is called the optimal control of problem (2.8), (2.9) with respect to the cost functional (2.11).

The aim of this paper is to study the asymptotic behavior, as $\varepsilon \rightarrow 0$ of the sequence of the optimal pairs $\left(u_{\varepsilon}, w_{\varepsilon}\right)$ under the following assumptions on the data:

$$
\begin{cases}\text { (i) } \quad & \widetilde{U}_{\varepsilon}^{0}-U^{0}:=\left(U_{1}^{0}, U_{2}^{0}\right) \quad \text { weakly in } L^{2}(\Omega) \times L^{2}(\Omega), \\ & \text { with } U_{2}^{0} \in H_{0}^{1}(\Omega), \\ \text { (ii) } & \widetilde{U}_{\varepsilon}^{1}-U^{1}:=\left(U_{1}^{1}, U_{2}^{1}\right) \quad \text { weakly in } L^{2}(\Omega) \times L^{2}(\Omega), \\ \text { (iii) } & \left\|U_{\varepsilon}^{0}\right\|_{H_{\gamma}^{\varepsilon} \leq C,}\end{cases}
$$

with $C$ positive constant independent of $\varepsilon$ and

$$
\widetilde{f}_{\varepsilon} \rightarrow f:=\left(f_{1}, f_{2}\right) \quad \text { weakly in } L^{2}\left(0, T ; L^{2}(\Omega)\right) \times L^{2}\left(0, T ; L^{2}(\Omega)\right)
$$

Let $A_{\gamma}^{0}$ be the matrix defined as follows: 
(i) for $\gamma<-1$

$$
A_{\gamma}^{0} \lambda=\frac{1}{|Y|} \int_{Y_{1}} A \nabla W_{\gamma} d y
$$

with $W_{\gamma} \in H^{1}\left(Y_{1}\right)$ a solution, for any $\lambda \in \mathbb{R}^{n}$, of

$$
\left\{\begin{array}{l}
-\operatorname{div}\left(A \nabla W_{\gamma}\right)=0 \quad \text { in } Y_{1} \\
W_{\gamma}-\lambda \cdot y \quad Y \text {-periodic } \\
\frac{1}{\left|Y_{1}\right|} \int_{Y_{1}}\left(W_{\gamma}-\lambda \cdot y\right) d y=0
\end{array}\right.
$$

(ii) for $\gamma=-1$

$$
A_{\gamma}^{0} \lambda=\frac{1}{|Y|} \int_{Y_{1}} A\left(\nabla \mathrm{w}^{1}+\nabla \mathrm{w}^{2}\right) d y
$$

with $\left(\mathrm{w}^{1}, \mathrm{w}^{2}\right) \in H^{1}\left(Y_{1}\right) \times H^{1}\left(Y_{2}\right)$ a solution, for any $\lambda \in \mathbb{R}^{n}$, of

$$
\left\{\begin{array}{l}
-\operatorname{div}\left(A \nabla \mathrm{w}^{1}\right)=0 \quad \text { in } Y_{1}, \\
-\operatorname{div}\left(A \nabla \mathrm{w}^{2}\right)=0 \quad \text { in } Y_{2}, \\
\left(A \nabla \mathrm{w}^{1}\right) \cdot n_{1}=-\left(A \nabla \mathrm{w}^{2}\right) \cdot n_{2} \quad \text { on } \Gamma, \\
-A \nabla \mathrm{w}^{1} \cdot n_{1}=h\left(\mathrm{w}^{1}-\mathrm{w}^{2}\right), \\
\mathrm{w}^{1}-\lambda \cdot y \quad Y \text {-periodic, } \\
\frac{1}{\left|Y_{1}\right|} \int_{Y_{1}}\left(\mathrm{w}^{1}-\lambda \cdot y\right) d y=0 ;
\end{array}\right.
$$

(iii) for $-1<\gamma<1$

$$
A_{\gamma}^{0} \lambda=\frac{1}{|Y|} \int_{Y_{1}} A \nabla \mathrm{w} d y
$$

with $\mathrm{w} \in H^{1}\left(Y_{1}\right)$ a solution, for any $\lambda \in \mathbb{R}^{n}$, of

$$
\left\{\begin{array}{l}
-\operatorname{div}(A \nabla \mathrm{w})=0 \quad \text { in } Y_{1} \\
(A \nabla \mathrm{w}) \cdot n_{1}=0 \quad \text { on } \Gamma \\
\mathrm{w}-\lambda \cdot y \quad Y \text {-periodic } \\
\frac{1}{\left|Y_{1}\right|} \int_{Y_{1}}(\mathrm{w}-\lambda \cdot y) d y=0
\end{array}\right.
$$

We establish the following result.

Theorem 2.1 Let $\gamma<1$ and let $A_{\varepsilon}$ and $h_{\varepsilon}$ satisfy (2.4)-(2.7). Suppose that (2.9), (2.13), and (2.14) hold and let $w_{\varepsilon}$ the optimal control of problem (2.8), (2.9), (2.11), and (2.12). Then there exists a function $w \in\left(L^{2}\left(0, T ; L^{2}(\Omega)\right)\right)^{2}$ such that

$$
\widetilde{w}_{\varepsilon} \rightarrow w=\left(w_{1}, w_{2}\right) \quad \text { in }\left(L^{2}\left(0, T ; L^{2}(\Omega)\right)\right)^{2},
$$

where

$$
w_{2}=\frac{\theta_{2}}{\theta_{1}} w_{1}
$$


and $\frac{w_{1}}{\theta_{1}}$ is the unique solution of the following problem:

$$
\min \left\{\frac{1}{2} \int_{0}^{T} \int_{\Omega}\left|u_{1}(z)\right|^{2}+\frac{1}{2} \int_{0}^{T} \int_{\Omega}|z|^{2}: z \in L^{2}\left(0, T ; L^{2}(\Omega)\right)\right\}
$$

$u_{1}(z)$ being, for every $z \in L^{2}\left(0, T ; L^{2}(\Omega)\right)$, the unique solution of

$$
\left\{\begin{array}{l}
\left.u_{1}^{\prime \prime}-\operatorname{div}\left(A_{\gamma}^{0} \nabla u_{1}\right)=f_{1}+f_{2}+z \quad \text { in } \Omega \times\right] 0, T[ \\
\left.u_{1}=0 \text { on } \partial \Omega \times\right] 0, T[ \\
u_{1}(0)=U_{1}^{0}+U_{2}^{0} \quad \text { in } \Omega \\
u_{1}^{\prime}(0)=U_{1}^{1}+U_{2}^{1} \quad \text { in } \Omega
\end{array}\right.
$$

where the homogenized matrix $A_{\gamma}^{0}$ is defined in (2.15), (2.17) and (2.19).

Remark 2.1 For $\gamma<-1$ the matrix field $A_{\gamma}^{0}$ is the classical one in a fixed domain. For $\gamma=$ -1 , the homogenized matrix $A_{\gamma}^{0}$ is described in terms of the periodic solution of an elliptic problem posed in the two reference sub-domains of the periodicity cell and prescribing on the interface a conormal derivative proportional to the jump of the solution. Finally in the case $-1<\gamma<1$, the matrix field $A_{\gamma}^{0}$ is the same obtained by Cioranescu and Saint Jean Paulin in [33], for the homogenization of the elliptic problem in the perforated domain $\Omega_{1 \varepsilon}$ with a Neumann condition on the boundary of the holes.

Remark 2.2 The boundness (iii) in (2.13) is necessary in order to have a priori estimates for the solution of problem (2.8) and (2.9), as shown in Section 3 below.

Remark 2.3 If $f_{i \varepsilon}=\left.f\right|_{\Omega_{\varepsilon i}}$, with $f \in L^{2}(\Omega)$, for $i=1,2$, then (2.14) holds with $f_{i}=\theta_{i} f$.

\section{Proof of Theorem 2.1}

This section is devoted to the proof of Theorem 2.1. At first, we recall some convergence results about the sequence of solutions of problem (2.8) and (2.9). Then we give a characterization of the optimal control $w_{\varepsilon}$ at $\varepsilon$-level in the form of the optimality system and we deduce a uniform estimate for $w_{\varepsilon}$. Finally we identify the limit of $w_{\varepsilon}$ as the solution of the optimality system related to the homogenized problem with respect to a suitable limit cost functional.

\subsection{Asymptotic behavior of the $\varepsilon$-problem}

Let $A_{\gamma}^{0}$ the matrix field defined in the previous section. We will make use of some homogenization results proved in [5] that we recall below, for the reader's convenience.

For any fixed $T>0$ and $\gamma<1$ let us consider the following problem:

$$
\left\{\begin{array}{l}
\left.u_{1 \varepsilon}^{\prime \prime}-\operatorname{div}\left(A^{\varepsilon} \nabla u_{1 \varepsilon}\right)=g_{1 \varepsilon} \quad \text { in } \Omega_{1 \varepsilon} \times\right] 0, T[, \\
\left.u_{2 \varepsilon}^{\prime \prime}-\operatorname{div}\left(A^{\varepsilon} \nabla u_{2 \varepsilon}\right)=g_{2 \varepsilon} \quad \text { in } \Omega_{2 \varepsilon} \times\right] 0, T[, \\
\left.A^{\varepsilon} \nabla u_{1 \varepsilon} \cdot n_{1 \varepsilon}=-A^{\varepsilon} \nabla u_{2 \varepsilon} \cdot n_{2 \varepsilon} \quad \text { on } \Gamma^{\varepsilon} \times\right] 0, T[, \\
\left.A^{\varepsilon} \nabla u_{1 \varepsilon} \cdot n_{1 \varepsilon}=-\varepsilon^{\gamma} h^{\varepsilon}\left(u_{1 \varepsilon}-u_{2 \varepsilon}\right) \quad \text { on } \Gamma^{\varepsilon} \times\right] 0, T[, \\
u_{1 \varepsilon}=0 \quad \text { on } \partial \Omega \times 0, T[, \\
u_{1 \varepsilon}(0)=U_{1 \varepsilon}^{0} \quad \text { in } \Omega_{1 \varepsilon}, \quad u_{2 \varepsilon}(0)=U_{2 \varepsilon}^{0} \quad \text { in } \Omega_{2 \varepsilon}, \\
u_{1 \varepsilon}^{\prime}(0)=U_{1 \varepsilon}^{1} \quad \text { in } \Omega_{1 \varepsilon}, \quad u_{2 \varepsilon}^{\prime}(0)=U_{2 \varepsilon}^{1} \quad \text { in } \Omega_{2 \varepsilon},
\end{array}\right.
$$


where $n_{i \varepsilon}$ is the unitary outward normal to $\Omega_{i \varepsilon}, i=1,2$, and

$$
\begin{cases}\text { (i) } & g_{\varepsilon}=\left(g_{1 \varepsilon}, g_{2 \varepsilon}\right) \in L^{2}\left(0, T ; L^{2}\left(\Omega_{1 \varepsilon}\right)\right) \times L^{2}\left(0, T ; L^{2}\left(\Omega_{2 \varepsilon}\right)\right) \\ \text { (ii) } & U_{\varepsilon}^{0}=\left(U_{1 \varepsilon}^{0}, U_{2 \varepsilon}^{0}\right) \in V^{\varepsilon} \times H^{1}\left(\Omega_{2 \varepsilon}\right) \\ \text { (iii) } & U_{\varepsilon}^{1}=\left(U_{1 \varepsilon}^{1}, U_{2 \varepsilon}^{1}\right) \in L^{2}\left(\Omega_{1 \varepsilon}\right) \times L^{2}\left(\Omega_{2 \varepsilon}\right)\end{cases}
$$

Moreover, let us suppose that

$$
\begin{cases}\text { (i) } & \widetilde{U}_{\varepsilon}^{0}-U^{0}:=\left(U_{1}^{0}, U_{2}^{0}\right) \quad \text { weakly in } L^{2}(\Omega) \times L^{2}(\Omega), \\ & \text { with } U_{2}^{0} \in H_{0}^{1}(\Omega), \\ \text { (ii) } \quad & \widetilde{U}_{\varepsilon}^{1}-U^{1}:=\left(U_{1}^{1}, U_{2}^{1}\right) \quad \text { weakly in } L^{2}(\Omega) \times L^{2}(\Omega), \\ \text { (iii) } \quad & \left\|U_{\varepsilon}^{0}\right\|_{H_{\gamma}^{\varepsilon}} \leq C,\end{cases}
$$

with $C$ positive constant independent of $\varepsilon$, and

$$
\widetilde{g_{\varepsilon}} \rightarrow g:=\left(g_{1}, g_{2}\right) \quad \text { weakly in } L^{2}\left(0, T ; L^{2}(\Omega)\right) \times L^{2}\left(0, T ; L^{2}(\Omega)\right) .
$$

Theorem 3.1 ([5]) Let $A_{\varepsilon}$ and $h_{\varepsilon}$ satisfy (2.4)-(2.7). Suppose that (3.2), (3.3), and (3.4) hold and let $u_{\varepsilon}$ be the solution of problem (3.1) and (3.2), with $\gamma<1$. Then there exists an extension operator $P_{1}^{\varepsilon} \in \mathcal{L}\left(L^{\infty}\left(0, T ; H^{k}\left(\Omega_{1 \varepsilon}\right)\right) ; L^{\infty}\left(0, T ; H^{k}(\Omega)\right)\right)$, for $k=1,2$, such that

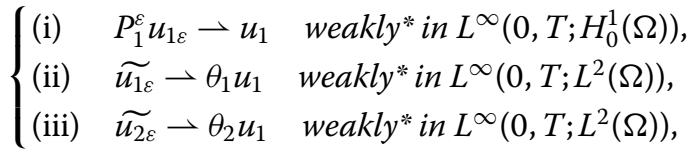

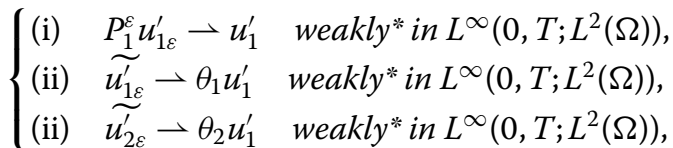

and

$$
A^{\varepsilon} \widetilde{\nabla u_{1 \varepsilon}}+A^{\varepsilon} \widetilde{\nabla u_{2 \varepsilon}} \rightarrow A_{\gamma}^{0} \nabla u_{1} \quad \text { weakly }{ }^{*} \text { in } L^{\infty}\left(0, T ;\left[L^{2}(\Omega)\right]^{n}\right)
$$

where $\theta_{1}$ and $\theta_{2}$ are given by (2.2) and $u_{1}$ is the unique solution in $L^{2}\left(0, T ; H_{0}^{1}(\Omega)\right)$, with $u_{1}^{\prime}$ in $L^{2}\left(0, T ; L^{2}(\Omega)\right)$, of the following problem:

$$
\left\{\begin{array}{l}
\left.u_{1}^{\prime \prime}-\operatorname{div}\left(A_{\gamma}^{0} \nabla u_{1}\right)=g_{1}+g_{2} \quad \text { in } \Omega \times\right] 0, T[ \\
\left.u_{1}=0 \text { on } \partial \Omega \times\right] 0, T[ \\
u_{1}(0)=U_{1}^{0}+U_{2}^{0} \quad \text { in } \Omega \\
u_{1}^{\prime}(0)=U_{1}^{1}+U_{2}^{1} \quad \text { in } \Omega .
\end{array}\right.
$$

Moreover, if $-1<\gamma<1$,

$$
\left\{\begin{array}{l}
\text { (i) } A^{\varepsilon} \widetilde{\nabla u_{1 \varepsilon}}-A_{\gamma}^{0} \nabla u_{1} \quad \text { weakly } \text { in }^{\infty}\left(0, T ;\left[L^{2}(\Omega)\right]^{n}\right), \\
\text { (ii) } A^{\varepsilon} \widetilde{\nabla u_{2 \varepsilon}} \rightarrow 0 \quad \text { weakly* in } L^{\infty}\left(0, T ;\left[L^{2}(\Omega)\right]^{n}\right) .
\end{array}\right.
$$

Let us recall the existence and uniqueness result, of the solution of problem (3.1) and (3.2), with $\gamma<1$ (see for instance [34], Theorem 24.A and [35], Chapter 3, Theorem 8.1, 
Remark 8.2). We point out that $H_{\gamma}^{\varepsilon} \subseteq L^{2}\left(\Omega_{1 \varepsilon}\right) \times L^{2}\left(\Omega_{2 \varepsilon}\right)$ with continuous imbedding so that the triple $\left(H_{\gamma}^{\varepsilon}, L^{2}\left(\Omega_{1 \varepsilon}\right) \times L^{2}\left(\Omega_{2 \varepsilon}\right), H_{\gamma}^{\varepsilon}\right)$ is an evolution triple (see Theorem 3.1 in [5] for an in-depth analysis on this aspect).

Theorem 3.2 Let $T \in] 0,+\infty\left[\right.$. Let $W_{\varepsilon}$ be defined as in (2.3), $h_{\varepsilon}$ and $A_{\varepsilon}$ as in (2.4)-(2.7). For every $\varepsilon$, under assumptions (3.2), (3.3), and (3.4), problem (3.1) admits a unique weak solution $u_{\varepsilon} \in W_{\varepsilon}$. Moreover, there exists a constant $C$, independent of $\varepsilon$, such that

$$
\begin{aligned}
& \left\|u_{\varepsilon}\right\|_{L^{2}\left(0, T H_{\gamma}^{\varepsilon}\left(\Omega_{\varepsilon}\right)\right)}+\left\|u_{\varepsilon}^{\prime}\right\|_{L^{2}\left(0, T L^{2}\left(\Omega_{1 \varepsilon}\right) \times L^{2}\left(\Omega_{2 \varepsilon}\right)\right)} \\
& \quad \leq C\left(\left\|U_{\varepsilon}^{0}\right\|_{H_{\gamma}^{\varepsilon}}+\left\|U_{\varepsilon}^{1}\right\|_{L^{2}\left(\Omega_{1 \varepsilon}\right) \times L^{2}\left(\Omega_{1 \varepsilon}\right)}+\left\|g_{\varepsilon}\right\|_{L^{2}\left(0, T ; L^{2}\left(\Omega_{1 \varepsilon}\right) \times L^{2}\left(\Omega_{2 \varepsilon}\right)\right)}\right) .
\end{aligned}
$$

Let us point out that, for any fixed $\varepsilon$, the solution of problem (3.1) and (3.2) has some further properties (see [35], Chapter 3, Theorem 8.2). In fact, under the same hypotheses of Theorem 3.2 the unique solution $u_{\varepsilon}$ of problem (3.1) and (3.2), with $\gamma<1$ satisfies

$$
u_{\varepsilon} \in C\left([0, T] ; H_{\gamma}^{\varepsilon}\right), \quad u_{\varepsilon}^{\prime} \in C\left([0, T] ; L^{2}\left(\Omega_{1 \varepsilon}\right) \times L^{2}\left(\Omega_{2 \varepsilon}\right)\right)
$$

and

$$
\begin{aligned}
& \left\|u_{\varepsilon}\right\|_{L^{\infty}\left(0, T H_{\gamma}^{\varepsilon}\left(\Omega_{\varepsilon}\right)\right)}+\left\|u_{\varepsilon}^{\prime}\right\|_{L^{\infty}\left(0, T L^{2}\left(\Omega_{1 \varepsilon}\right) \times L^{2}\left(\Omega_{2 \varepsilon}\right)\right)} \\
& \quad \leq C\left(\left\|U_{\varepsilon}^{0}\right\|_{H_{\gamma}^{\varepsilon}}+\left\|U_{\varepsilon}^{1}\right\|_{L^{2}\left(\Omega_{1 \varepsilon}\right) \times L^{2}\left(\Omega_{1 \varepsilon}\right)}+\left\|g_{\varepsilon}\right\|_{L^{2}\left(0, T ; L^{2}\left(\Omega_{1 \varepsilon}\right) \times L^{2}\left(\Omega_{2 \varepsilon}\right)\right)}\right),
\end{aligned}
$$

where $C$ is the same constant as in (3.10).

\subsection{The optimality system}

The following results give a characterization of the optimal controls for both problem at level $\varepsilon$ and homogenized problem (2.24) (see [11], Chapter 4).

Theorem 3.3 For every $\varepsilon$, under assumptions (2.4)-(2.7) and (2.9), the optimal pair $\left(u_{\varepsilon}, w_{\varepsilon}\right)$, solution of problem (2.8), (2.9), (2.11), and (2.12) is characterized by the following optimality system:

$$
\begin{aligned}
& \left\{\begin{array}{l}
\left.u_{1 \varepsilon}^{\prime \prime}-\operatorname{div}\left(A^{\varepsilon} \nabla u_{1 \varepsilon}\right)=f_{1 \varepsilon}+w_{1 \varepsilon} \quad \text { in } \Omega_{1 \varepsilon} \times\right] 0, T[, \\
\left.u_{2 \varepsilon}^{\prime \prime}-\operatorname{div}\left(A^{\varepsilon} \nabla u_{2 \varepsilon}\right)=f_{2 \varepsilon}+w_{2 \varepsilon} \quad \text { in } \Omega_{2 \varepsilon} \times\right] 0, T[, \\
\left.A^{\varepsilon} \nabla u_{1 \varepsilon} \cdot n_{1 \varepsilon}=-A^{\varepsilon} \nabla u_{2 \varepsilon} \cdot n_{2 \varepsilon} \quad \text { on } \Gamma^{\varepsilon} \times\right] 0, T[, \\
\left.A^{\varepsilon} \nabla u_{1 \varepsilon} \cdot n_{1 \varepsilon}=-\varepsilon^{\gamma} h^{\varepsilon}\left(u_{1 \varepsilon}-u_{2 \varepsilon}\right) \quad \text { on } \Gamma^{\varepsilon} \times\right] 0, T[, \\
\left.u_{1 \varepsilon}=0 \quad \text { on } \partial \Omega \times\right] 0, T[, \\
u_{1 \varepsilon}(0)=U_{1 \varepsilon}^{0} \quad \text { in } \Omega_{1 \varepsilon}, \quad u_{2 \varepsilon}(0)=U_{2 \varepsilon}^{0} \quad \text { in } \Omega_{2 \varepsilon}, \\
u_{1 \varepsilon}^{\prime}(0)=U_{1 \varepsilon}^{1} \quad \text { in } \Omega_{1 \varepsilon}, \quad u_{2 \varepsilon}^{\prime}(0)=U_{2 \varepsilon}^{1} \quad \text { in } \Omega_{2 \varepsilon},
\end{array}\right. \\
& \begin{cases}\left.p_{1 \varepsilon}^{\prime \prime}-\operatorname{div}\left(A^{\varepsilon} \nabla p_{1 \varepsilon}\right)=u_{1 \varepsilon} \quad \text { in } \Omega_{1 \varepsilon} \times\right] 0, T[, \\
\left.p_{2 \varepsilon}^{\prime \prime}-\operatorname{div}\left(A^{\varepsilon} \nabla p_{2 \varepsilon}\right)=u_{2 \varepsilon} \quad \text { in } \Omega_{2 \varepsilon} \times\right] 0, T[, \\
\left.A^{\varepsilon} \nabla p_{1 \varepsilon} \cdot n_{1 \varepsilon}=-A^{\varepsilon} \nabla p_{2 \varepsilon} \cdot n_{2 \varepsilon} \quad \text { on } \Gamma^{\varepsilon} \times\right] 0, T[, \\
\left.A^{\varepsilon} \nabla p_{1 \varepsilon} \cdot n_{1 \varepsilon}=-\varepsilon^{\gamma} h^{\varepsilon}\left(p_{1 \varepsilon}-p_{2 \varepsilon}\right) \quad \text { on } \Gamma^{\varepsilon} \times\right] 0, T[, \\
\left.p_{1 \varepsilon}=0 \quad \text { on } \partial \Omega \times\right] 0, T[, \\
p_{1 \varepsilon}(T)=0 \quad \text { in } \Omega_{1 \varepsilon}, \quad p_{2 \varepsilon}(T)=0 \quad \text { in } \Omega_{2 \varepsilon}, \\
p_{1 \varepsilon}^{\prime}(T)=0 \quad \text { in } \Omega_{1 \varepsilon}, \quad p_{2 \varepsilon}^{\prime}(T)=0 \quad \text { in } \Omega_{2 \varepsilon},\end{cases}
\end{aligned}
$$




$$
\left.p_{\varepsilon}=-w_{\varepsilon} \text { a.e. } \operatorname{in}\right] 0, T\left[\times \Omega_{\varepsilon} .\right.
$$

As previously, we prefer to use the following weak formulation of problems (3.13) and (3.14):

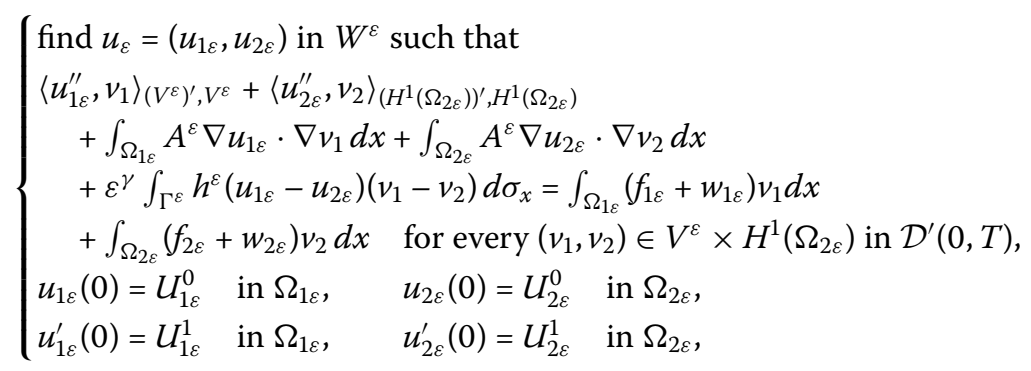

and

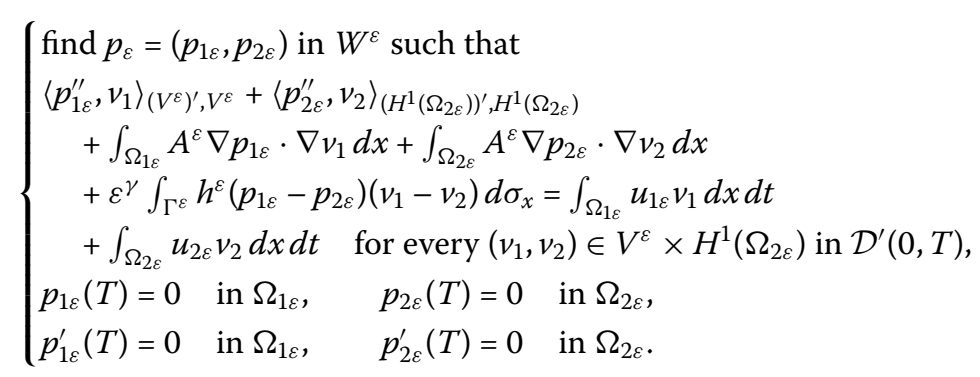

Let us consider the cost functional $J_{\infty}: L^{2}\left(0, T ; L^{2}(\Omega)\right) \rightarrow \mathbb{R}$ defined in the following way:

$$
J_{\infty}(z):=\frac{1}{2} \int_{0}^{T} \int_{\Omega}\left|u_{1}(z)\right|^{2}+\frac{1}{2} \int_{0}^{T} \int_{\Omega}|z|^{2}
$$

where for every control $z \in L^{2}\left(0, T ; L^{2}(\Omega)\right), u_{1}(z)$ is the unique solution of problem (2.24). This functional is continuous, strictly convex, and coercive. Hence, by applying the direct method in the calculus of variations, the minimum problem (2.23) admits a unique solution $\bar{w}$ which is the optimal control of problem (2.24) with respect to the cost functional (3.18).

Theorem 3.4 The optimal pair $\left(u_{1}, \bar{w}\right)$, solution of problem (2.23) and (2.24) is characterized by the following optimality system:

$$
\begin{aligned}
& \left\{\begin{array}{l}
\left.u_{1}^{\prime \prime}-\operatorname{div}\left(A_{\gamma}^{0} \nabla u_{1}\right)=f_{1}+f_{2}+\bar{w} \quad \text { in } \Omega \times\right] 0, T[ \\
\left.u_{1}=0 \text { on } \partial \Omega \times\right] 0, T[ \\
u_{1}(0)=U_{1}^{0}+U_{2}^{0} \quad \text { in } \Omega, \\
u_{1}^{\prime}(0)=U_{1}^{1}+U_{2}^{1} \quad \text { in } \Omega,
\end{array}\right. \\
& \left\{\begin{array}{l}
\left.p_{1}^{\prime \prime}-\operatorname{div}\left(A_{\gamma}^{0} \nabla p_{1}\right)=u_{1} \quad \text { in } \Omega \times\right] 0, T[ \\
\left.u_{1}=0 \quad \text { on } \partial \Omega \times\right] 0, T[ \\
p_{1}(T)=0 \quad \text { in } \Omega, \\
p_{1}^{\prime}(T)=0 \text { in } \Omega,
\end{array}\right. \\
& p_{1}=-\bar{w} .
\end{aligned}
$$




\subsection{A priori estimates}

In this subsection, we deduce some a priori norm-estimates either for the sequence of the optimal controls $w_{\varepsilon}$ or for the corresponding solution $u_{\varepsilon}=u_{\varepsilon}\left(w_{\varepsilon}\right)$ (resp. $p_{\varepsilon}=p_{\varepsilon}\left(u_{\varepsilon}\right)$ ) of problem (3.16) (resp. (3.17)).

Proposition 3.1 Let $\left(u_{\varepsilon}, w_{\varepsilon}\right) \in\left(L^{2}\left(0, T ; L^{2}\left(\Omega_{1 \varepsilon}\right) \times L^{2}\left(\Omega_{2 \varepsilon}\right)\right)\right)^{2}$ be the optimal pair, solution of the optimality system (3.13), (3.14) and (3.15). Under assumptions (2.4)-(2.7), (2.13) and (2.14), there exists a constant $c$, independent of $\varepsilon$, such that

$$
\begin{gathered}
\left\|w_{\varepsilon}\right\|_{L^{2}\left(0, T ; L^{2}\left(\Omega_{1 \varepsilon}\right) \times L^{2}\left(\Omega_{2 \varepsilon}\right)\right)} \leq c, \\
\left\|u_{\varepsilon}\right\|_{L^{2}\left(0, T ; L^{2}\left(\Omega_{1 \varepsilon}\right) \times L^{2}\left(\Omega_{2 \varepsilon}\right)\right)} \leq c,
\end{gathered}
$$

for every $\varepsilon$.

Proof Let us fix $\varepsilon$. Let $u_{\varepsilon}=u_{\varepsilon}\left(w_{\varepsilon}\right)$ be the unique solution of problem (3.16) and $p_{\varepsilon}=p_{\varepsilon}\left(u_{\varepsilon}\right)$ be the unique solution of the adjoint problem (3.17). Choosing $p_{\varepsilon}$ as test function in (3.16) and $u_{\varepsilon}$ as a test function in (3.17), we have

$$
\begin{aligned}
\int_{0}^{T} & \left\langle u_{1 \varepsilon}^{\prime \prime}(t, \cdot), p_{1 \varepsilon}(t, \cdot)\right\rangle_{\left(H^{1}\left(\Omega_{1 \varepsilon}\right)\right), H^{1}\left(\Omega_{1 \varepsilon}\right)} d t+\int_{0}^{T}\left\langle u_{2 \varepsilon}^{\prime \prime}(t, \cdot), p_{2 \varepsilon}(t, \cdot)\right\rangle_{\left(H^{1}\left(\Omega_{2 \varepsilon}\right)\right), H^{1}\left(\Omega_{2 \varepsilon}\right)} d t \\
\quad & \quad \int_{0}^{T} \int_{\Omega_{1 \varepsilon}} A \nabla_{x} u_{1 \varepsilon} \nabla_{x} p_{1 \varepsilon}+u_{1 \varepsilon} p_{1 \varepsilon} d x d t+\int_{0}^{T} \int_{\Omega_{2 \varepsilon}} A \nabla_{x} u_{2 \varepsilon} \nabla_{x} p_{2 \varepsilon}+u_{2 \varepsilon} p_{2 \varepsilon} d x d t \\
& =\int_{0}^{T} \int_{\Omega_{1 \varepsilon}} f_{\varepsilon} p_{1 \varepsilon}+w_{1 \varepsilon} p_{1 \varepsilon} d x d t+\int_{0}^{T} \int_{\Omega_{2 \varepsilon}} f_{\varepsilon} p_{2 \varepsilon}+w_{2 \varepsilon} p_{2 \varepsilon} d x d t
\end{aligned}
$$

and

$$
\begin{aligned}
& \int_{0}^{T}\left\langle p_{1 \varepsilon}^{\prime \prime}(t, \cdot), u_{1 \varepsilon}(t, \cdot)\right\rangle_{\left(H^{1}\left(\Omega_{1 \varepsilon}\right)\right), H^{1}\left(\Omega_{1 \varepsilon}\right)} d t+\int_{0}^{T}\left\langle p_{2 \varepsilon}^{\prime \prime}(t, \cdot), u_{2 \varepsilon}(t, \cdot)\right\rangle_{\left(H^{1}\left(\Omega_{2 \varepsilon}\right)\right), H^{1}\left(\Omega_{2 \varepsilon}\right)} d t \\
& \quad+\int_{0}^{T} \int_{\Omega_{1 \varepsilon}} A \nabla_{x} p_{1 \varepsilon} \nabla_{x} u_{1 \varepsilon}+p_{1 \varepsilon} u_{1 \varepsilon} d x d t+\int_{0}^{T} \int_{\Omega_{2 \varepsilon}} A \nabla_{x} p_{2 \varepsilon} \nabla_{x} u_{2 \varepsilon}+p_{2 \varepsilon} u_{2 \varepsilon} d x d t \\
& \quad=\int_{0}^{T} \int_{\Omega_{1 \varepsilon}}\left(u_{1 \varepsilon}\right)^{2} d x d t+\int_{0}^{T} \int_{\Omega_{2 \varepsilon}}\left(u_{2 \varepsilon}\right)^{2} d x d t
\end{aligned}
$$

Integrating by parts, we get

$$
\begin{aligned}
\int_{0}^{T} & \left\langle u_{1 \varepsilon}^{\prime \prime}(t, \cdot), p_{1 \varepsilon}(t, \cdot)\right\rangle_{\left(H^{1}\left(\Omega_{1 \varepsilon}\right)\right)^{\prime}, H^{1}\left(\Omega_{1 \varepsilon}\right)} d t+\int_{0}^{T}\left\langle u_{1 \varepsilon}^{\prime \prime}(t, \cdot), p_{1 \varepsilon}(t, \cdot)\right\rangle_{\left(H^{1}\left(\Omega_{1 \varepsilon}\right)\right)^{\prime}, H^{1}\left(\Omega_{1 \varepsilon}\right)} d t \\
= & \left\langle u_{1 \varepsilon}^{\prime}(T), p_{1 \varepsilon}(T)\right\rangle_{\left(H^{1}\left(\Omega_{1 \varepsilon}\right)\right)^{\prime}, H^{1}\left(\Omega_{1 \varepsilon}\right)}-\left\langle u_{1 \varepsilon}^{\prime}(0), p_{1 \varepsilon}(0)\right\rangle_{\left(H^{1}\left(\Omega_{1 \varepsilon}\right)\right)^{\prime}, H^{1}\left(\Omega_{1 \varepsilon}\right)} \\
& -\int_{0}^{T}\left(u_{1 \varepsilon}^{\prime}(t, \cdot), p_{1 \varepsilon}^{\prime}(t, \cdot)\right)_{L^{2}\left(\Omega_{1 \varepsilon}\right)} d t+\left\langle u_{2 \varepsilon}^{\prime}(T), p_{2 \varepsilon}(T)\right\rangle_{\left(H^{1}\left(\Omega_{2 \varepsilon}\right)\right)^{\prime}, H^{1}\left(\Omega_{2 \varepsilon}\right)} \\
& -\left\langle u_{2 \varepsilon}^{\prime}(0), p_{2 \varepsilon}(0)\right\rangle_{\left(H^{1}\left(\Omega_{2 \varepsilon}\right)\right)^{\prime}, H^{1}\left(\Omega_{2 \varepsilon}\right)}-\int_{0}^{T}\left(u_{2 \varepsilon}^{\prime}(t, \cdot), p_{2 \varepsilon}^{\prime}(t, \cdot)\right)_{L^{2}\left(\Omega_{2 \varepsilon}\right)} d t
\end{aligned}
$$


and

$$
\begin{aligned}
\int_{0}^{T}\left\langle p_{1 \varepsilon}^{\prime \prime}(t, \cdot), u_{1 \varepsilon}(t, \cdot)\right\rangle_{\left(H^{1}\left(\Omega_{1 \varepsilon}\right)\right)^{\prime}, H^{1}\left(\Omega_{1 \varepsilon}\right)} d t+\int_{0}^{T}\left\langle p_{1 \varepsilon}^{\prime \prime}(t, \cdot), u_{1 \varepsilon}(t, \cdot)\right\rangle_{\left(H^{1}\left(\Omega_{1 \varepsilon}\right)\right)^{\prime}, H^{1}\left(\Omega_{1 \varepsilon}\right)} d t \\
=\left\langle p_{1 \varepsilon}^{\prime}(T), u_{1 \varepsilon}(T)\right\rangle_{\left(H^{1}\left(\Omega_{1 \varepsilon}\right)\right)^{\prime}, H^{1}\left(\Omega_{1 \varepsilon}\right)}-\left\langle p_{1 \varepsilon}^{\prime}(0), u_{1 \varepsilon}(0)\right\rangle_{\left(H^{1}\left(\Omega_{1 \varepsilon}\right)\right)^{\prime}, H^{1}\left(\Omega_{1 \varepsilon}\right)} \\
\quad-\int_{0}^{T}\left(p_{1 \varepsilon}^{\prime}(t, \cdot), u_{1 \varepsilon}^{\prime}(t, \cdot)\right)_{L^{2}\left(\Omega_{1 \varepsilon}\right)} d t+\left\langle p_{2 \varepsilon}^{\prime}(T), u_{2 \varepsilon}(T)\right\rangle_{\left(H^{1}\left(\Omega_{2 \varepsilon}\right)\right)^{\prime}, H^{1}\left(\Omega_{2 \varepsilon}\right)} \\
-\left\langle p_{2 \varepsilon}^{\prime}(0), u_{2 \varepsilon}(0)\right\rangle_{\left(H^{1}\left(\Omega_{2 \varepsilon}\right)\right)^{\prime}, H^{1}\left(\Omega_{2 \varepsilon}\right)}-\int_{0}^{T}\left(p_{2 \varepsilon}^{\prime}(t, \cdot), u_{2 \varepsilon}^{\prime}(t, \cdot)\right)_{L^{2}\left(\Omega_{2 \varepsilon}\right)} d t
\end{aligned}
$$

Subtracting (3.24) from (3.25), using (3.26) and (3.27), the symmetry of the matrix $A$, the initial conditions in (3.16), and the final conditions in (3.17), we obtain

$$
\begin{aligned}
& \left\langle p_{1 \varepsilon}^{\prime}(0), U_{1 \varepsilon}^{0}\right\rangle_{\left(H^{1}\left(\Omega_{1 \varepsilon}\right)\right)^{\prime}, H^{1}\left(\Omega_{1 \varepsilon}\right)}-\left\langle U_{1 \varepsilon}^{1}, p_{1 \varepsilon}(0)\right\rangle_{\left(H^{1}\left(\Omega_{1 \varepsilon}\right)\right)^{\prime}, H^{1}\left(\Omega_{1 \varepsilon}\right)} \\
& \quad+\left\langle p_{2 \varepsilon}^{\prime}(0), U_{2 \varepsilon}^{0}\right\rangle_{\left(H^{1}\left(\Omega_{2 \varepsilon}\right)\right)^{\prime}, H^{1}\left(\Omega_{2 \varepsilon}\right)}-\left\langle U_{2 \varepsilon}^{1}, p_{2 \varepsilon}(0)\right\rangle_{\left(H^{1}\left(\Omega_{2 \varepsilon}\right)\right)^{\prime}, H^{1}\left(\Omega_{2 \varepsilon}\right)} \\
& =\int_{0}^{T} \int_{\Omega_{1 \varepsilon}} f_{1 \varepsilon} p_{1 \varepsilon}+w_{1 \varepsilon} p_{1 \varepsilon}-u_{1 \varepsilon}^{2} d x d t+\int_{0}^{T} \int_{\Omega_{2 \varepsilon}} f_{2 \varepsilon} p_{2 \varepsilon}+w_{2 \varepsilon} p_{2 \varepsilon}-u_{2 \varepsilon}^{2} d x d t .
\end{aligned}
$$

By virtue of (3.15), as a result we find

$$
\begin{aligned}
\int_{0}^{T} & \int_{\Omega_{1 \varepsilon}} u_{1 \varepsilon}^{2}+w_{1 \varepsilon}^{2} d x d t+\int_{0}^{T} \int_{\Omega_{2 \varepsilon}} u_{2 \varepsilon}^{2}+w_{2 \varepsilon}^{2} d x d t \\
= & -\int_{0}^{T} \int_{\Omega_{1 \varepsilon}} f_{1 \varepsilon} w_{1 \varepsilon} d x d t-\int_{0}^{T} \int_{\Omega_{2 \varepsilon}} f_{2 \varepsilon} w_{2 \varepsilon} d x d t \\
& +\int_{\Omega_{1 \varepsilon}} U_{1 \varepsilon}^{1} p_{1 \varepsilon}(0)-U_{1 \varepsilon}^{0} p_{1 \varepsilon}^{\prime}(0) d x+\int_{\Omega_{2 \varepsilon}} U_{2 \varepsilon}^{1} p_{2 \varepsilon}(0)-U_{2 \varepsilon}^{0} p_{2 \varepsilon}^{\prime}(0) d x
\end{aligned}
$$

from which, by the Cauchy-Schwarz inequality, it follows that

$$
\begin{aligned}
\left\|u_{\varepsilon}\right\|_{L^{2}\left(0, T ; L^{2}\left(\Omega_{1 \varepsilon}\right) \times L^{2}\left(\Omega_{2 \varepsilon}\right)\right)}^{2}+\left\|w_{\varepsilon}\right\|_{L^{2}\left(0, T ; L^{2}\left(\Omega_{1 \varepsilon}\right) \times L^{2}\left(\Omega_{2 \varepsilon}\right)\right)}^{2} \\
\leq\left\|w_{\varepsilon}\right\|_{L^{2}\left(0, T ; L^{2}\left(\Omega_{1 \varepsilon}\right) \times L^{2}\left(\Omega_{2 \varepsilon}\right)\right)}\left\|f_{\varepsilon}\right\|_{L^{2}\left(0, T ; L^{2}\left(\Omega_{1 \varepsilon}\right) \times L^{2}\left(\Omega_{2 \varepsilon}\right)\right)} \\
\quad+\left\|U_{\varepsilon}^{0}\right\|_{L^{2}\left(\Omega_{1 \varepsilon}\right) \times L^{2}\left(\Omega_{2 \varepsilon}\right)}\left\|p_{\varepsilon}^{\prime}(0, \cdot)\right\|_{L^{2}\left(\Omega_{1 \varepsilon}\right) \times L^{2}\left(\Omega_{2 \varepsilon}\right)} \\
\quad+\left\|U_{\varepsilon}^{1}\right\|_{L^{2}\left(\Omega_{1 \varepsilon}\right) \times L^{2}\left(\Omega_{2 \varepsilon}\right)}\left\|p_{\varepsilon}(0, \cdot)\right\|_{L^{2}\left(\Omega_{1 \varepsilon}\right) \times L^{2}\left(\Omega_{2 \varepsilon}\right)} .
\end{aligned}
$$

On the other hand, to estimate $\left\|p_{\varepsilon}(0, \cdot)\right\|_{L^{2}\left(\Omega_{1 \varepsilon}\right) \times L^{2}\left(\Omega_{2 \varepsilon}\right)}$ and $\left\|p_{\varepsilon}^{\prime}(0, \cdot)\right\|_{L^{2}\left(\Omega_{1 \varepsilon}\right) \times L^{2}\left(\Omega_{2 \varepsilon}\right)}$ let us apply Theorem 3.2 with $p_{\varepsilon}$ instead of $u_{\varepsilon}$. Then by translation we get

$$
\left\|p_{\varepsilon}(0, \cdot)\right\|_{H^{1}\left(\Omega_{\varepsilon}\right)}+\left\|p_{\varepsilon}^{\prime}(0, \cdot)\right\|_{L^{2}\left(\Omega_{\varepsilon}\right)} \leq C\left\|u_{\varepsilon}\right\|_{L^{2}\left(0, T ; L^{2}\left(\Omega_{1 \varepsilon}\right) \times L^{2}\left(\Omega_{2 \varepsilon}\right)\right)}
$$

where $C$ is a constant independent of $\varepsilon$. Finally, combining (3.29) with (3.30) and using (2.13), as a result we find that

$$
\begin{aligned}
& \left\|u_{\varepsilon}\right\|_{L^{2}\left(0, T ; L^{2}\left(\Omega_{1 \varepsilon}\right) \times L^{2}\left(\Omega_{2 \varepsilon}\right)\right)}^{2}+\left\|w_{\varepsilon}\right\|_{L^{2}\left(0, T ; L^{2}\left(\Omega_{1 \varepsilon}\right) \times L^{2}\left(\Omega_{2 \varepsilon}\right)\right)}^{2} \\
& \quad \leq c_{3}\left\|w_{\varepsilon}\right\|_{L^{2}\left(0, T ; L^{2}\left(\Omega_{1 \varepsilon}\right) \times L^{2}\left(\Omega_{2 \varepsilon}\right)\right)}+c_{4}\left\|u_{\varepsilon}\right\|_{L^{2}\left(0, T ; L^{2}\left(\Omega_{1 \varepsilon}\right) \times L^{2}\left(\Omega_{2 \varepsilon}\right)\right)},
\end{aligned}
$$

with constants independent of $\varepsilon$. So estimates (3.22) and (3.23) are proved. 
As a consequence of (3.22), up to a subsequence still denoted by $\varepsilon$, we have the following convergences:

$$
\begin{array}{ll}
\widetilde{w_{1 \varepsilon}} \rightarrow w_{1} & \text { in } L^{2}\left(0, T ; L^{2}(\Omega)\right), \\
\widetilde{w_{2 \varepsilon}} \rightarrow w_{2} & \text { in } L^{2}\left(0, T ; L^{2}(\Omega)\right) .
\end{array}
$$

\subsection{Conclusions}

Let us recall that for the adjoint state at $\varepsilon$-level $p_{\varepsilon}=\left(p_{1 \varepsilon}, p_{2 \varepsilon}\right)$, the following convergences hold:

$$
\begin{aligned}
& \widetilde{p_{1 \varepsilon}} \rightarrow \theta_{1} p_{1} \quad \text { weakly* in } L^{\infty}\left(0, T ; L^{2}(\Omega)\right), \\
& \widetilde{p_{2 \varepsilon}} \rightarrow \theta_{2} p_{1} \quad \text { weakly* in } L^{\infty}\left(0, T ; L^{2}(\Omega)\right) .
\end{aligned}
$$

Hence by (3.15) and (3.32) we get

$$
\begin{aligned}
& \theta_{1} p_{1}=-w_{1}, \\
& \theta_{2} p_{1}=-w_{2},
\end{aligned}
$$

where $\theta_{1}$ and $\theta_{2}$ are given in (2.2). As a consequence

$$
w_{2}=\frac{\theta_{2}}{\theta_{1}} w_{1}
$$

which is (2.22).

Hence we are able to pass to the limit, as $\varepsilon$ goes to 0 , in the optimality system (3.13)-(3.15) by applying Theorem 3.1 to both problems (3.13) and (3.14) with, respectively, $g_{\varepsilon}=f_{\varepsilon}+w_{\varepsilon}$ and $g_{\varepsilon}=u_{\varepsilon}$. Then by (2.14), (3.32), and (3.33) as $\theta_{1}+\theta_{2}=1$, we see that the pair $\left(u_{1}, \frac{w_{1}}{\theta_{1}}\right)$ is such that

$$
\begin{aligned}
& \left\{\begin{array}{l}
\left.u_{1}^{\prime \prime}-\operatorname{div}\left(A_{\gamma}^{0} \nabla u_{1}\right)=f_{1}+f_{2}+\frac{1}{\theta_{1}} w_{1} \quad \text { in } \Omega \times\right] 0, T[, \\
\left.u_{1}=0 \text { on } \partial \Omega \times\right] 0, T[, \\
u_{1}(0)=U_{1}^{0}+U_{2}^{0} \text { in } \Omega, \\
u_{1}^{\prime}(0)=U_{1}^{1}+U_{2}^{1} \text { in } \Omega,
\end{array}\right. \\
& \left\{\begin{array}{l}
\left.p_{1}^{\prime \prime}-\operatorname{div}\left(A_{\gamma}^{0} \nabla p_{1}\right)=\theta_{1} u_{1}+\theta_{2} u_{1}=u_{1} \quad \text { in } \Omega \times\right] 0, T[ \\
\left.u_{1}=0 \text { on } \partial \Omega \times\right] 0, T[, \\
p_{1}(T)=0 \text { in } \Omega, \\
p_{1}^{\prime}(T)=0 \text { in } \Omega,
\end{array}\right. \\
& p_{1}=-\frac{1}{\theta_{1}} w_{1} .
\end{aligned}
$$

Finally, by Theorem 3.4 and uniqueness we get $\frac{w_{1}}{\theta_{1}}=\bar{w}$ and the convergences (3.5)-(3.7) and (3.32) hold for the whole sequence. Hence Theorem 2.1 is now completely proved. 


\section{Authors' contributions}

The authors conceived and wrote this article in collaboration and with the same responsibility. All of them read and approved the final manuscript.

\section{Author details}

'Dipartimento di Ingegneria Elettrica e dell' Informazione, Università degli Studi di Cassino e del Lazio Meridionale, via G. Di Biasio 43, Cassino, FR 03043, Italia. ${ }^{2}$ Dipartimento di Scienze e Tecnologie, Universitá del Sannio, Via Dei Mulini 59/A Palazzo Inarcassa, Benevento, 82100, Italia.

Received: 17 November 2014 Accepted: 4 March 2015 Published online: 17 March 2015

\section{References}

1. Auriault, JL, Ene, $\mathrm{H}$ : Macroscopic modelling of heat transfer in composites with interfacial thermal barrier. Int. J. Heat Mass Transf. 37, 2885-2892 (1994)

2. Canon, E, Pernin, JN: Homogenization of diffusion in composite media with interfacial barrier. Rev. Roum. Math. Pures Appl. 44, 23-36 (1999)

3. Donato, P: Some corrector results for composites with imperfect interface. Rend. Mat. Appl. (7) 26, 189-209 (2006)

4. Donato, P: Homogenization of a class of imperfect transmission problems. In: Damlamian, A, Miara, B, Li, T (eds.) Multiscale Problems: Theory, Numerical Approximation and Applications. Series in Contemporary Applied Mathematics CAM, vol. 16, pp. 109-147. Higher Education Press, Beijing (2011)

5. Donato, P, Faella, L, Monsurrò, S: Homogenization of the wave equation in composites with imperfect interface: a memory effect. J. Math. Pures Appl. 87, 119-143 (2007)

6. Donato, P, Faella, L, Monsurrò, S: Correctors for the homogenization of a class of hyperbolic equations with imperfect interfaces. SIAM J. Math. Anal. 40, 1952-1978 (2009)

7. Donato, $\mathrm{P}$, Jose, E: Corrector results for a parabolic problem with a memory effect. ESAIM: Math. Model. Numer. Anal. 44, 421-454 (2010)

8. Donato, $\mathrm{P}$, Jose, E: Asymptotic behavior of the approximate controls for parabolic equations with interfacial contact resistance. ESAIM Control Optim. Calc. Var. 21, 138-164 (2015). doi:10.1051/cocv/2014029

9. Donato, P, Monsurrò, S: Homogenization of two heat conductors with interfacial contact resistance. Anal. Appl. 2, 247-273 (2004)

10. Monsurrò, S: Erratum for the paper 'Homogenization of a two-component composite with interfacial thermal barrier'. Adv. Math. Sci. Appl. 14, 375-377 (2004)

11. Lions, JL: Optimal Control of System Governed by Partial Differential Equations. Springer, Berlin (1971)

12. Hummel, HC: Homogenization for heat transfer in polycrystals with interfacial resistances. Appl. Anal. 75, 403-424 (2000)

13. Monsurrò, S: Homogenization of a two-component composite with interfacial thermal barrier. Adv. Math. Sci. Appl. $13,43-63(2003)$

14. De Maio, U, Gaudiello, A, Lefter, C: Optimal control for a parabolic problem in a domain with highly oscillating boundary. Appl. Anal. 83(12), 1245-1264 (2004)

15. De Maio, U, Faella, L, Perugia, C: Optimal control problem for an anisotropic parabolic problem in a domain with very rough boundary. Ric. Mat. 63(2), 307-328 (2014)

16. De Maio, U, Faella, L, Perugia, C: Optimal control for a second-order linear evolution problem in a domain with oscillating boundary. Complex Var. Elliptic Equ. doi:10.1080/17476933.2015.1022169 (to appear)

17. De Maio, U, Nandakumaran, AK, Perugia, C: Exact internal controllability for the wave equation in a domain with oscillating boundary with Neumann boundary condition. Evol. Equ. Control Theory (2015, to appear)

18. Durante, T, Faella, L, Perugia, C: Homogenization and behaviour of optimal controls for the wave equation in domains with oscillating boundary. NoDEA Nonlinear Differ. Equ. Appl. 14(5-6), 455-489 (2007)

19. Durante, T, Mel'nyk, TA: Asymptotic analysis of an optimal control problem involving a thick two-level junction with alternate type of controls. J. Optim. Theory Appl. 144(2), 205-225 (2010)

20. Durante, T, Mel'nyk, TA: Homogenization of quasilinear optimal control problems involving a thick multilevel junction of type 3:2:1. ESAIM Control Optim. Calc. Var. 18(2), 583-610 (2012)

21. Lions, JL: Contrôlabilité Exacte et Homogénéisation. I. Asymptot. Anal. 1(1), 3-11 (1988)

22. Cioranescu, D, Donato, P: Exact internal controllability in perforated domains. J. Math. Pures Appl. 68(2), 185-213 (1989)

23. Cioranescu, D, Donato, P, Zuazua, E: Exact boundary controllability for the wave equation in domains with small holes. J. Math. Pures Appl. 71(4), 343-377 (1992)

24. Donato, P, Nabil, A: Homogenization and correctors for the heat equation in perforated domains. Ric. Mat. 50(1), 115-144 (2001)

25. Faella, L, Perugia, C: Homogenization of a Ginzburg-Landau problem in a perforated domain with mixed boundary conditions. Bound. Value Probl. 2014, 223 (2014). doi:10.1186/s13661-014-0223-2

26. Lipton, R: Heat conduction in fine scale mixtures with interfacial contact resistance. SIAM J. Appl. Math. 58, 55-72 (1998)

27. Lipton, R, Vernescu, B: Composite with imperfect interface. Proc. R. Soc. Lond. Ser. A 452, 329-358 (1996)

28. Monsurrò, S: Homogenization of a composite with very small inclusions and imperfect interface. In: Multi Scale Problems and Asymptotic Analysis. GAKUTO Internat. Ser. Math. Sci. Appl., vol. 24, pp. 217-232. Gakkōtosho, Tokyo (2006)

29. Yang, Z: Homogenization and correctors for the hyperbolic problems with imperfect interfaces via the periodic unfolding method. Commun. Pure Appl. Anal. 13(1), 249-272 (2014)

30. Yang, Z: The periodic unfolding method for a class of parabolic problems with imperfect interfaces. ESAIM: Math Model. Numer. Anal. 48(5), 1279-1302 (2014)

31. Cioranescu, D, Donato, P: An Introduction to Homogenization. Oxford Lecture Ser. Math. Appl., vol. 17. Oxford University Press, New York (1999) 
32. Faella, L, Monsurrò, S: Memory effects arising in the homogenization of composites with inclusions. In: Topics on Mathematics for Smart System, pp. 107-121. World Scientific, Hackensack (2007)

33. Cioranescu, D, Saint Jean Paulin, J: Homogenization in open sets with holes. J. Math. Anal. Appl. 71, 590-607 (1979)

34. Zeidler, E: Nonlinear Functional Analysis and Its Applications. Vol II, Part A and B. Springer, Berlin (1980)

35. Lions, JL, Magenes, E: Non-Homogeneous Boundary Value Problems and Applications, vol. 1. Springer, Berlin (1972)

Submit your manuscript to a SpringerOpen ${ }^{\odot}$ journal and benefit from:

- Convenient online submission

- Rigorous peer review

- Immediate publication on acceptance

- Open access: articles freely available online

- High visibility within the field

- Retaining the copyright to your article

Submit your next manuscript at $\boldsymbol{s p r i n g e r o p e n . c o m ~}$ 